\title{
LOS PILARES ESENCIALES PARA UNA EDUCACIÓN INCLUSIVA DE LAS PERSONAS HOMOSEXUALES
}

\author{
THE ESSENTIAL PILLARS FOR AN INCLUSIVE EDUCATION OF HOMOSEXUAL PEOPLE
}

\author{
Douglas Verbicaro Soares
}

Universidade Federal de Roraima, Boa Vista, RR, Brasil. E-mail: douglas_verbicaro@yahoo.com.br

\section{Maria Clara Govêia de Oliveira}

Universidade Federal de Roraima, Boa Vista, RR, Brasil. E-mail: mariaclarinha.rr@gmail.com

\author{
Jhemylly Wilhena Alves
}

Universidade Federal de Roraima, Boa Vista, RR, Brasil. E-mail: jwilhenaa@gmail.com

DOI: https://doi.org/10.46550/amormundi.v2i3.110

Recebido em: 29.07.2021

Aceito em: 18.08.2021

\begin{abstract}
Resumen: Dentro de la sociedad brasileña, el prejuicio hacia las personas homosexuales sigue siendo muy fuerte, por lo que todavía hay mucho repudio social dentro de los institutos más básicos de la sociedad. En el conocimiento de las desventajas de tener un desarrollo en medio de tales condiciones, este artículo busca explorar los pilares esenciales de la educación, con el fin de entender: ¿Cómo puede llegar a ser más inclusivo y acogedor para las personas homosexuales? Utilizando el método deductivo y la investigación bibliográfica, se discute el pilar familiar y se hacen consideraciones sobre la familia y la homosexualidad, además del pilar escolar. Por último, se concluye por la necesidad de una acción estatal para fomentar una educación que garantice la dignidad humana, los derechos fundamentales y el desarrollo físico, mental y social de las personas homosexuales.
\end{abstract}

Palabras clave: Igualdad. Discriminación. Orientación sexual.

Abstract: Within Brazilian society, prejudice towards gay people is still very strong, so there is still much social repudiation within the most basic institutes of society. In understanding the disadvantages of having a development during such conditions, this article seeks to explore the essential pillars of education, to understand: How can it become more inclusive and welcoming to gay people? Using the deductive method and bibliographical research, the family pillar is discussed, and considerations are made about the family and homosexuality, in addition to the school pillar. Finally, it concludes with the need for state action to promote education that guarantees human dignity, fundamental rights, and the physical, mental, and social development of homosexual people.

Keywords: Equality. Discrimination. Sexual preference. 


\section{Introducción}

Zldesarrollo de una persona está impregnado de varias fases. En estos diversos momentos, las experiencias e influencias que se están experimentando forman pensamiento, personalidad y, entre otros factores, que hacen que una persona sea única. El entorno familiar, igualmente con el entorno escolar, son donde se producen los primeros contactos con otras personas y, así, se aprende a vivir en sociedad.

Sin embargo, este desarrollo no es pacífico para todos en realidad, particularmente para las personas homosexuales. La familia puede convertirse en un contacto muy incómodo a medida que se desarrolla, incluso siendo inexistente. Además, la escuela puede ser un lugar traumático, el escenario de los peores recuerdos de una persona.

En conocimiento de esto, este estudio tiene como objetivo discutir: ¿Cómo la educación puede ser más inclusiva y aceptada para las personas homosexuales a través de pilares básicos: la familia y la escuela? Además, pretendemos debatir sobre la importancia de estos dos institutos y las consecuencias de este tipo de educación en la sociedad. De acuerdo con la metodología utilizada en el artículo, se utilizó un enfoque teórico cualitativo, basado en la investigación bibliográfica multidisciplinar.

Para ello, en este artículo se aborda un tema para hablar del pilar de la familia, otro para hablar de su relación con la homosexualidad y otro para hablar del pilar de la escuela. Por último, se hacen las últimas consideraciones y conclusiones, en las que se considera el papel de los poderes públicos en la búsqueda de una educación más inclusiva y acogedora para las personas homosexuales.

\section{El pilar de la familia}

Siguiendo esa línea de raciocinio, para alcanzar un ambiente de mayor aceptación e integración de la diversidad sexual en la sociedad brasileña, se hace necesaria enfocar esa educación en los pilares basilares de esa sociedad. Por esta razón, hemos apuntado la familia, como el primer pilar, que se representa por medio de una institución social de una persona, donde se desarrollan los primeros aprendizajes. También es la organización responsable de la formación del perfil de cada persona.

Para In`am Al Mufti el papel fundamental de la institución, de la familia se hace indispensable para permitir la descubierta y de desarrollar las aptitudes individuales de cada persona. No representa una tarea única de la escuela. El papel de la familia en una sensibilización de sus miembros puede fomentar la participación inclusiva de todos sus miembros en sociedad (AL MUFTI, 1997, p. 223). Palabras que estamos de acuerdo como un instrumento de desarrollo y de liberación responsable para una ciudadanía participativa.

De ésta importante institución surge la preocupación en constituirse familias estructuradas, con ambientes familiares saludables para sus integrantes y preparadas para ofertar una educación de modo consciente a sus integrantes (JORNAL DO FEDERAL, 2009, p. 10-3). Esta institución representa el primer contacto de una persona con el mundo social, los primeros descubrimientos, las experiencias iniciales se desarrollan en este ambiente organizado, es decir, dentro del medio familiar. Por esta razón, es fundamental la consolidación de una 
estructura familiar ordenada para permitir el pleno desarrollo físico y mental de sus individuos (VERBICARO SOARES, 2012, p. 51-2).

De acuerdo con Fraile González:

Retomando el papel de la familia, nos planteamos si los valores, las reglas, los ritos familiares...contribuyen a la estabilidad familiar, si funcionan como sello de identidad para las distintas familias. Por otro lado, existen fuerzas internas y externas, como es el proceso evolutivo de los miembros de una familia, los conflictos, las crisis que funcionan como agentes de cambio. El equilibrio entre ambas fuerzas contribuirá al crecimiento y estabilidad de la familia.

El autor defiende el posicionamiento del denominado modelo evolutivo-educativo, teniendo en cuenta algunos pilares principales: a) la formación de un ambiente de encuentro entre distintas generaciones, b) la constitución de un vínculo de apoyo para administrar los problemas enfrentados en el seno de la familia, c) un ambiente de desarrollo para los miembros de la familia, d) la formación de un proyecto de vida común por medio de una educación compartida (FRAILE GONZÁLEZ, 2008, p. 55).

Sobre el tema de la sexualidad, la familia ya previamente mencionada es la responsable de la formación de la esencia del comportamiento humano, junto con otras instituciones (escuela, religión, trabajo), que van a orientar la creación de los perfiles humanos y las maneras de relacionarse unos con los otros (FRAILE GONZÁLEZ, 2008, p. 55).

Al desarrollar esa investigación, hemos encontrado las consideraciones de Charlotte Pátterson que nos aclaró la difícil tarea de los vínculos familiares en la aceptación de las personas homosexuales, comparándola como un verdadero caos el reconocimiento de esa orientación sexual en el ámbito familiar. La realidad, con los tiempos, había condicionado la homosexualidad a una característica negativa, estudios, opiniones de profesionales de la salud, tajaran de incorrecta o antinatural las conductas afectivas entre las personas del mismo sexo biológico.

De este modo, las relaciones entre padres e hijos han sido, en muchos de los casos, basada en estereotipos peyorativos hacia a las personas homosexuales. Por un lado los hijos que descubrían su sexualidad, pero que se sentían atemorizados en asumirla para sus padres o familiares por el miedo de sufrir rechazo. Y los padres por otro lado, al encontrarse con un caso de homosexualidad en la familia, luchaban para negarlo y peor, de buscar medios para cambiar o supuestamente curar, la homosexualidad de sus hijos. En este sentido, la relación basada en el respeto de los hijos hacia a sus padres y la visión de autoridad que los primeros tendrían, lo hacía más complicado para un hijo homosexual asumirme en el seno familiar, con el miedo de rechazo por los ascendentes.

Hemos encontrado un relato en la ciudad de Belém, en la provincia brasileña de Pará, donde un hijo homosexual nos ha revelado que se acuerda de un momento clave en su vida, cuando su padre, un policía civil jubilado, le presionó a tomar un rumbo en su vida dándole un plazo de un mes para que su hijo presentarse en casa con una novia, caso contrario debería dejar la residencia familiar, pues no quería tener un hijo homosexual. Hecho ese que nos ha llamado la atención, una vez que consideramos el pilar familiar como un eje fundamental para auxiliar y apoyar a las personas a desarrollasen plenamente en dignidad. Al depararnos con este caso, podemos evaluar que problemas de este tipo pueden repercutir de modo negativa en la vida de una persona, pues no aceptarse como es, quizás sea uno de los retos más difíciles de 
llevar consigo, pues sería como actuar y no ser correcto consigo mismo, viviendo una falsa vida, aparentando algo que no lo es.

Podemos citar que también era homosexual uno de los líderes de los menores asesinados cruelmente en el episodio conocido mundialmente en la Iglesia de la Candelaria en 1993, en la ciudad carioca de Río de Janeiro, en la provincia de Río de Janeiro en Brasil. Episodio que terminó con la muerte de 8 (ocho) personas sin hogar en la ciudad, seis de ellos eran menores y fueran muertos por policías militares. Los casos de violencia a los homosexuales no cesan, pues, también, encontramos relatos de prácticas realizadas por familiares en la provincia de Bahía, que se aportan desde la época de la esclavitud de Brasil colonia, cuando a un familiar cercano tomaba conocimiento del desarrollo de la orientación sexual homosexual de un descendente, como forma de castigo, hacía una limpieza anal en estas personas con pimienta (MOTT, 1997, p. 19).

Mott nos complementa la idea con la evidencia de diversos casos en que las personas homosexuales acaban siendo obligadas a abandonar sus familias por los más distintos motivos. Para el autor: unos han escapado de casa, otros han sido expulsados, del ámbito familiar, por no resistieren a la intolerancia doméstica. Hay casos en que el suicido acaba siendo la "solución" para tanta opresión. Entre algunos relatos citamos a los amantes homosexuales, que más cercano a la obra ficticia shakespeariana de Romeo y Julieta, han decidido poner fin a sus vidas de modo pactado, los dos primos pertenecientes a una familia con mucho dinero en la ciudad de Salvador, en la provincia de Bahia en Brasil. Los dos jóvenes han dejado una nota culpando la extrema opresión familiar como la causa justificadora del suicidio de los integrantes de la familia Bruni (MOTT, 1997, p. 19).

Por otro lado, en las últimas décadas, cada vez más, los hijos a aceptaren y de entender su propia integridad sexual han dado pasos a reconocer frente a sus familiares su orientación sexual en edades más tempranas, siendo el lugar donde viven un influyente en este camino hacia el reconocimiento, es decir, las localidades más pequeñas, los hijos tienden a no revelar su sexualidad de modo temprano, por otro lado en ciudades mayores, es paso se da de modo menos reluctante.

Soriano Rubio nos aclara que: quizás los elementos que se deban valorar sean: si para el propio bienestar es importante que lo sepan, si se está preparado para decírselo, cuáles son las características de la familia (edad, creencias morales, actitudes) y cómo es la relación con ellos (SORIANO RUBIO, 1999, p. 187-9).

Los temas de la dependencia financiera también pueden influir en el despertar y el reconocimiento de la sexualidad de un joven en el ámbito de la familia. Las personas que van a estudiar fuera y viven otras realidades, tienden a asumirse más tempranamente, pues consiguen estar fuera de un ambiente cerrado y conservador de la mentalidad de un pueblo conservador, hecho que destaca los centros urbanos como emancipadores de descubiertas a la diversidad existente sobre la sexualidad humana, cuanto más amplia, abierta, multicultural se hace el ambiente, más fácil será el asumir personal de una persona sobre su sexualidad (PATTERSON, 1998, p. 94-5).

Para López Sánchez, cuando resalta el papel de los padres que influyen de manera más importante en los aspectos relacionados con sus hijos. Ellos son los que tienen el derecho y el deber de transmitir a sus hijos el conocimiento, la educación, las actitudes, valores, normas y también, en los campos de las relaciones interpersonales y sexuales esta función de ayudar a 
consolidar los valores (LÓPEZ SÁNCHEZ, 2005, p. 163-4).

Según las explanaciones sobre el tema en portugués:

Os pais são os que influenciam de forma mais importante os filhos em muitos aspectos, entre eles, em todas as aprendizagens relacionadas com os afetos e a sexualidade. Mesmo que os pais não falem abertamente desses temas, transmitem o mais importante para a educação sexual: relaçôes confiadas ou desconfiadas, afetuosas ou distantes, igualitárias ou discriminatórias, ideias positivas sobre o mundo, o ser humano e a vida ou ideias hostis, desesperançosas (LÓPEZ SÁNCHEZ, 2005, p. 163-4).

Lo que esto representa: que los padres tienen importante influencia en la vida de los hijos, es decir, toda la enseñanza en temas de afectos y sexualidad, por más que hablar de estos temas no sean fáciles, los padres transmiten directa o indirectamente sus valores, sus modales, ideas, comportamientos, afecto, tanto en temas de igualdad o desigualdad, lo que repercutirá futuramente en las actitudes de sus hijos en el ámbito privado (doméstico) y en el ámbito público en sociedad (LÓPEZ SÁNCHEZ, 2005, p. 163-4).

Es en este sentido que se añade que los padres, así como otros responsables, que también pueden ocupar esa función primordial, cuando se tenga la ausencia estas figuras serán los abuelos, los tíos, con grados de parentesco sanguíneos o no, que podrán desarrollar, a través de sus enseñanzas, las validas contribuciones en la vida de sus dependientes.

\section{Reflexiones sobre la familia y la homosexualidad}

A lo largo de la realización de este estudio, cuando hemos estado hablando sobre la homosexualidad, hemos notado que el desconocimiento mayoritario sobre la materia, hace que se forme una afirmación impropia, como por ejemplo: de que las personas homosexuales serán incapaces de constituir familias sólidas, o que la formación de una unidad familiar entre personas del mismo sexo representaría unos hechos no naturales (EL SEXO DE LOS DINOSAURIOS, 2011), representando tanto un riesgo para los niños de padres homosexuales, incluso para la sociedad (ROCHA, 2014).

De acuerdo con Consuelo Jávega:

La homosexualidad, la bisexualidad y la transexualidad han sido, históricamente, objeto de rechazo por gran parte de la sociedad, rechazo que puede llegar a manifestarse a través de conductas discriminatorias que tienen una indudable transcendencia desde el punto de vista jurídico en las diversas disciplinas del Derecho. Tradicionalmente, se ha presentado a la familia desde el núcleo de convivencia en el matrimonio entre heterosexuales, desde la perspectiva de la finalidad reproductora del mismo, negándose a nivel social y jurídico otras realidades afectivas, $\mathrm{o}$, simplemente, silenciándolas de forma consciente e intencionada. Sin embargo, este concepto de familia resulta día a día más caduco, con la aparición de nuevas fórmulas de convivencia que reclaman abiertamente sus espacios de libertad y su correlativo reconocimiento a nivel jurídico (CHACARTEGUI JÁVEGA, 2001, p. 23).

La historia nos ha visibilizado numerosas conductas discriminatorias hacia a los colectivos supra mencionados, poniéndoles en una situación de exclusión participativa y social en las distintas sociedades por el mundo, a lo largo de los tiempos. Estos hechos negativos hicieron de la homosexualidad, por ejemplo, un concepto negativo y de fuerte carácter peyorativo, que atribuía a las personas que tuviesen esa orientación sexual una connotación prejudicial de promiscuidad, 
de poca moral, persona que no se pudiese tener confianza y otras más.

Eses argumentos reflejaran en la definición general antigua de quelas personas homosexuales eran incapaces de constituir familia, sea en tener una pareja estable o de poder educar a un hijo. Incapacidad esa reflejada en la supuesta incapacidad de los padres homosexualidad en la creación de sus hijos ha sido incluso, objeto de manifestación en el sistema interamericano, de la Corte Interamericana de Derecho Humanos, caso Jacqueline Karen Atala Riffo verso Chile, caso ese mencionado en nuestro trabajo, lo que demostró que el tema de la homosexualidad sigue siendo muy controvertido, pero que ya existe una comprensión tanto en diversos países en sus derechos fundamentales, como en el plan internacional en el reconocimiento de derechos a las personas homosexuales.

Contra el posicionamiento de la CIDH, nos hemos deparado con diversos comentarios. Un ejemplo de ese posicionamiento está en el ámbito español de Pedro Sanz, perteneciente al Partido Popular de la Rioja, que se pronunció en su momento con la siguiente idea: un niño en una unión de parejas del mismo sexo creo que no tiene las mismas oportunidades, que no tiene una educación exactamente igual que un niño que nace dentro de una familia, o de un matrimonio, o de un entorno de parejas heterosexuales (VERBICARO SOARES, 2012, p. 53).

Para estos comentarios, se ha manifestado de modo diverso la diputada del Partido Socialista Español, de la misma comunidad autonómica, Inmaculada Ortega. Para la representante política:

Sanz nos pone nuevamente en el punto de mira de toda Espańa, dando una imagen retrógrada y neoconservadora de nuestra tierra cuando sus afirmaciones no las compartimos una gran parte de la sociedad riojana. La diputada también ańadió que: los niños lo que necesitan en sus hogares es afecto, equilibrio, seguridad, normas compartidas y eso lo pueden dar todas las personas de la familia independientemente de su condición sexual (VERBICARO SOARES, 2012, p. 53).

En el ámbito social, de personas que consideraban a las parejas de homosexuales incapaces de engendrar o adoptar nińos, pues esta condición representaría un grave problema para el proceso educativo de una crianza en el medio social, como si la condición de ser homosexual imposibilitase la propagación de una cultura educativa pertinente y necesaria para la integridad física y mental de otra persona. De esta manera se explica las relaciones de género y la homosexualidad, simples cómo son. En su condición de existencia esencial, la subordinación rígida de su supuesta incapacidad de añadir a la sociedad, su especificación de ser distinto, diferente, de tener sus propias ventajas, como ocurre con la predominancia del género masculino sobre el femenino y la orientación del deseo heterosexual sobre los demás - homosexual o bisexual (VERBICARO SOARES, 2012, p. 53).

Quizás unos de las opiniones más polémicas de los últimos años en Brasil ha sido de la política Marina Silva, en la época candidata a las elecciones a la presidencia de la República brasileña, en 2010. Se recuerda que la candidata se posicionó en contra a la adopción públicamente de niños por personas homosexuales, generando en el país toda una discusión sobre la temática de los derechos del colectivo LGBT. Más actualmente, en 2014, la política Silva volvió a presentarse como candidata a la presidencia, teniendo que rectificar su posicionamiento con relación a su postura, en este momento, reconociendo la posibilidad de la adopción a los homosexuales.

En una entrevista publicada en día 1 de octubre de 2014, la periodista Christiane 
Amanpour (CNN), Silva había comentado que en su programa de Gobierno había sido el que mejor, entre los demás presentados por otros candidatos, que mejor contaba con propuestas para los homosexuales. Es válido recordar que la discusión más reciente con relación a las propuestas de Marina Silva ha sido un cambio de postura de la candidata, al retirar de su programa electoral temas relacionados a los derechos de las personas homosexuales, como por ejemplo: la no criminalización de la homofobia y la legalización del casamiento civil entre personal del mismo sexo (ESTADÃO, 2014).

Para contradecir el argumento de la supuesta incapacidad de creación de niños por padres homosexual, hemos optado citar la existencia de estudios para la verificación de las repercusiones en la vida de los hijos creados por parejas del mismo sexo (homosexuales) se han encontrado resultados que determinan que los niños de esos padres no tienen problemas generados por esta condición, es decir, que las personas que justifican ese imposibilidad, tienen contrapuestos argumentos científicos que atestan justo lo contrario.

En España nos hemos deparado, en este trabajo, con comentarios que los niños pueden tener un desarrollo favorable a ser creados basados en el reconocimiento, respeto y la igualdad de trato entre las personas. Estas conclusiones extraídas de un estudio solicitado por la Fiscalía del Menor de Madrid - España, a través de Javier Urra, psicólogo. De acuerdo con las aportaciones del profesional y, también en estudios recomendado de psicólogos de Madrid y Andalucía, indagando lo que ocurría con los padres homosexuales en tener hijos, apuntaban que no se encontraban nada significativo que demostrara la imposibilidad de padres homosexuales la adopción niños (DYKERAMA, 2010).

Del mismo modo en que la Asociación de Psicólogos Americana, en un ideario conjunto defendido por López Sánchez, se posicionan en los temas de adopción por personas homosexuales que la orientación sexual de una persona no afecta en el pleno desarrollo de un niño, una vez que para el correcto desarrollo necesita es afecto y amor (ALDER IZQUIERDO, 2013, p. 239).

Estamos completamente de acuerdo que la creación de un ambiente saludable basado por el amor que los padres exteriorizan hacia a sus hijos, sean de padres homosexuales o heterosexual, que lo importante es la demostración de ese afecto, por lo tanto, las razones que se presentan en el sentido contrario a esa posibilidad no parecen a nuestro entendimiento, plausibles de fundamentos, una vez que estudios científicos y la opinión de profesionales ya han se manifestado a favor de las adopciones de niños por padres con una orientación sexual homosexual o bisexual, que ese factor no incide de modo negativo en la creación de los niños.

Podemos citar más uno entre distintos estudios sobre la temática abordada, referente a evaluar las repercusiones en la vida, precisamente sobre los hijos creados por parejas del mismo sexo. Para la pesquisidora Nanette Gartrell, que ha acompañado 78 parejas y ha avaluado el bienestar de los hijos a través de cuestionarios, entrevistas. Las respuestas han sido codificadas, sometidas al análisis y posteriormente comparadas con los datos de crianzas de parejas heterosexuales.

Según este estudio, pues todavía son pocos los que abordan la temática homosexual, que tuvo su inicio en el año de 1986, ha revelado que los niños educados por parejas de lesbianas tienen mayor equilibrio psicológico y presentan menos problemas de comportamiento. Hecho este que pueden demonstrar que la orientación del deseo homosexual no es en sí, un problema para la creación, educación de los individuos (hijos) en sociedad, por lo contrario, pueden añadir importantes ensañamientos y a ayudar a combatir las injusticias y auxiliar en el proceso 
de integración social. Para la $\mathrm{CNN}$, hijos de parejas de homosexuales revelaran competencias superiores en niveles sociales, académicos y menor tendencia para problemas de agresividad e incumplimiento de reglas (DYKERAMA, 2010).

De este modo, es válido recordar que para evitar las desigualdades de trato puede estar en la educación por el respeto al próximo, aceptar lo diverso, lo diferente, buscar el equilibrio en la educación familiar y que sea posible una relación más armónica dentro y fuera de los vínculos familiares (JORNAL DO FEDERAL, 2009, p. 10-3). Con base en esta argumentación, podemos demonstrar que en Brasil, algunas iniciativas se han desarrollado para evidenciar e integrar socialmente el tema de la orientación sexual en el ámbito de la enseñanza, como por ejemplo algunas normas específicas de algunos municipios brasileños, que buscan la educación para la igualdad, son ellas: Ley n. ${ }^{\circ}$ 3.582/1998 de Campo Grande/ MS: que habla de la educación llevando en consideración la orientación sexual y el planteamiento familiar a los padres y alumnos del preescuela y $1^{\circ}$ Grado de estudios (primarias) en la red municipal de enseñanza; Ley n. ${ }^{\circ}$ 12.284/2006 de São Paulo/SP, que autoriza el Poder Ejecutivo a incluir, en el currículo de la enseñanza fundamental y media, los preceptos de críticas a la violencia familiar, de género y los de discriminación por raza, orientación sexual; Ley n. ${ }^{\circ}$ 12.491/1997 de Minas Gerais /MG, que determina la inclusión del contenido y actividades para la orientación sexual en la grade curricular de la enseñanza fundamental de este Estado (CEZÁRIO, 2007, p. 61-5).

\section{El pilar de la escuela}

Es válido informar que otro pilar para la integración de la diversidad se plasma en la figura de la escuela, el conjunto base de la enseñanza, es allí donde ocurre la complementación de la educación recibida en casa, a través de la familia. La escuela tiene el objetivo de integrar a las personas en el ambiente de las relaciones personales, tanto dentro de las familias, como en el mundo que la rodea, por lo tanto, tiene una función complementaria (LÓPEZ SÁNCHEZ, 2006, p. 164). En este contexto, el trabajo para educar en la igualdad, tratando los temas de género y los de la sexualidad humana, educando en el modo responsable de la aceptación de todos socialmente, sin discriminación, permitiendo una convivencia armónica con la igualdad de trato entre todas las personas (VERBICARO SOARES, 2012, p. 55-6).

No nos cabe duda de que el proyecto representa una iniciativa válida para el proceso de concienciación social sobre la temática de la orientación del deseo y, consecuentemente, para la sexualidad y los derechos sexuales como los derechos humanos.

Nuestro planteamiento se basa en la conciencia colectiva de la importancia de la movilidad de la sociedad brasileña, por medio de sus sectores, entre ellos las escuelas, pilares indispensables para la formación ciudadana y la transmisión de valores cívicos y democráticos, dentro de las casas familiares, en el trabajo y otras esferas. Por esa razón son instrumentos materializados para la formación de opinión de los propios ciudadanos del país.

Aduciremos que los cobros por cambios, cuando un Estado muestra flaqueza cuanto a la implementación de políticas públicas en el sentido de fortalecer la educación y de motiva a la participación ciudadana, tendrá la sociedad otras alternativas para cambiar las realidades. Iniciativa esa que podría ser seguida por el Gobierno brasileño, así como la sociedad en general (sociedad civil, empresas públicas) en la aplicación de esas medidas para facilitar la inclusión 
social de las personas homosexuales.

Indispensables para la elucidación de los problemas visibilizados cuanto al tema de la educación son los comentarios de West, que al analizar algunos casos de políticas implementadas en el ámbito internacional para la educación, por ejemplo a de la Conferencia en el año de 1990 de Tailandia, se constató que el problema no estaba solamente en la idea de muchos Estados u Organizaciones de la ONU, con el apoyo de entidades locales, nacionales e internacionales en la potenciación de los recursos destinados a educación.

El autor, de modo oportuno recuerda que, lo importante no sería solamente los recursos destinados, pero sí la determinación de las verdaderas necesidades para el sector, como bien aclara el autor: la principal prioridad consiste en determinar, antes de gastar más (G. WEST, 1994, p. 13). Del mismo modo que West enfatiza que: las nociones de igualdad y de igualdad de oportunidades merecen un tratamiento muy cuidadoso (G. WEST, 1994, p. 273).

Estamos de acuerdo con ese ideal presentado. A veces la solución más simples para el combate del problema educacional no está en la captación exacerbada de recursos, pero la apropiada aplicación de los mismos en estrategia previamente ordenadas y pensadas de modo racional previamente, para no caer en conductas, muy conocidas en Brasil, los casos de corrupción general del Gobierno, donde los recursos supuestamente destinados a la educación son constantemente materia de cortes presupuestarios o de apropiación indebida por los representantes de los ciudadanos a favor de intereses particulares.

\section{Consideraciones finales}

Por lo tanto, a la vista de lo presentado, este trabajo permitió un análisis más profundo de los pilares esenciales para una educación inclusiva de las personas homosexuales y, en el curso de este estudio, dos instituciones demostraron ser fundamentales en la formación de la esencia, la personalidad y la educación: la familia y la escuela. Dado que los primeros rasgos de personalidad, así como las ideas, creencias, religión y el pleno desarrollo físico y mental de un ser humano se desarrollarán dentro de la familia, de modo que esta estructura permite los contactos sociales primarios de un individuo y ejerce una gran influencia en su vida y relaciones interpersonales. La escuela, en lo que a ella respecta, se une a la estructura familiar para que el individuo tenga pleno éxito en su educación, integrando y transmitiendo los valores esenciales para una experiencia social armoniosa.

Sin embargo, la estructura familiar no siempre está presente para los homosexuales, lo que genera inseguridad sobre la sexualidad de estos individuos y causa consecuencias en su vida y educación. En este sentido, la escuela tiene gran relevancia en la acogida de estas personas, lo que se puede lograr a través de la promoción de la igualdad y la conciencia sobre los derechos sexuales individuales. En Brasil, en algunos estados, ya existen medidas que buscan implementar acciones para estimular la igualdad y el respeto en la escuela. Sin embargo, todavía se han adoptado pocas medidas en relación con los homosexuales y el entorno escolar.

Por lo tanto, las autoridades públicas brasileñas deben fomentar más acciones dirigidas a una educación inclusiva que no sólo integre a los homosexuales en el cuerpo social, sino que fomente la aceptación de la familia y la comunidad cuando no ocurre a través de la lucha de puntos de vista peyorativos sobre la homosexualidad, para que tal unión entre la comunidad, la 
familia y la escuela pueda ofrecer a los individuos homosexuales la estructura educativa necesaria y esencial para la garantía de la dignidad humana. , los derechos fundamentales y el desarrollo físico, mental y social total de estas personas.

\section{Referencias}

ALDER IZQUIERDO, Alejandro. Realidad jurídica y social del derecho a la orientación e identidad de género. Tesis Doctoral. Universidad de Salamanca: Programa de Doctorado Pasado y presente de los derechos humanos, Salamanca, España, 2013.

AL MUFTI, IN`AM: "La excelencia en la educación: hay que invertir en el talento" en: VVAA.: La educación encierra un tesoro. México, DF, México: Ediciones UNESCO, 1997.

CEZÁRIO, Joelma. Legislação e Jurisprudência LGBTTT. Instituto de Bioética, Direitos Humanos e Gênero e Associação Lésbica Feminista de Brasília Coturno de Vênus, Brasília, Brasil, 2007.

CHACARTEGUI JÁVEGA, Consuelo. Discriminación y orientación sexual del trabajador. $1^{\mathrm{a}}$ Edición. Valladolid, España: Editorial Lex Nova, 2001.

Documentario Español: El sexo de los dinosaurios. Disponible en: <http://www. elsexodelosdinosaurios.com/>. Acceso en: 10 de julio de 2021.

DYKERAMA. Filhos de lésbicas são mais equilibrados, diz estudo. 7 junio de 2010. Disponible en: <http://dykerama.uol.com.br/src/?mI=5\&cID=23\&iID=3145\&nome=Filhos_ de_1\%E9sbicas_s\%E3o_mais_equilibrados,_diz_estudo>. Acceso en: 10 de julio de 2021.

ESTADÃO. Fui a que melhor integrou propostas aos gays, diz Marina. Disponible en: <https:// br.noticias.yahoo.com/fui-melhor-integrou-propostas-aos-gays-diz-marina-145300130.html>. Acceso en: 10 de julio de 2021.

FRAILE GONZÁLEZ, Eduardo. "La educación em la familia del medio rural: igualdad de oportunidades". In: MAYA FRADES, Valentina: Mujeres Rurales. Estudios multidisciplinares de género. Ediciones Universidad de Salamanca. Salamanca, España, 2008.

G. WEST, Edwin. La educación y el Estado: Um estúdio de conomía política. Madrid, España: Unión Editorial, 1994.

JORNAL DO FEDERAL. Antigas lutas insistem em novos rumos para a sociedade. Conselho Federal de Psicologia - Ano XXI no 93 - Junho, Brasília, Brasil, 2009.

LÓPEZ SÁNCHEZ, Félix. Homosexualidad y familia. Lo que los padres, madres, homosexuales y profesionales deben saber y hacer. 1a Edición. Barcelona, España: Editorial GRAÓ, 2006.

LÓPEZ SÁNCHEZ, Félix. La Educación Sexual. Madrid, España: Biblioteca Nueva, 2005.

MOTT, Luiz Roberto. Homofobia. Aviolção dos Direitos Humanos de Gays, Lésbicas \& Travestis no Brasil. Grupo Gay Da Bahia / International Gay and Lesbian Human Rights Commission. Salvador. Bahia, Brasil, 1997.

PATTERSON, Charlotte. Lesbian, gay, and bisexual identities in families: psychological perspectives. Oxford University Press, Oxford, New York. United States, 1998. 
ROCHA, Alexander. El presidente popular de La Rioja ha criticado la crianza de niños por parte de parejas homosexuales. Disponible en: <http://noticias.universogay.com/el-presidente-popularde-la-rioja-ha-criticado-la-crianza-de-ninos-por-parte-de-parejas-homosexuales_20022012. html>. Acceso en: 21 de julio 2021.

SORIANO RUBIO, Sonia. Como se vive la homosexualidad y el lesbianismo. Salamanca, España: Amarú Ediciones, 1999.

VERBICARO SOARES, Douglas. Las mujeres y las personas homosexuales en las Fuerzas Armadas. Especial referencia a las FFAA brasileñas. Trabajo fin de curso Máster Oficial en Estudios Interdisciplinares de Género. Universidad de Salamanca. Salamanca, España, 2012. 